\title{
FERTILIZACIÓN NITROGENADA EN EL CRECIMIENTO, CONTENIDO DE COMPUESTOS FENÓLICOS Y ACTIVIDAD ANTIOXIDANTE DE ALBAHACA
}

\section{NITROGEN FERTILIZATION EFFECTS ON GROWTH, PHENOLIC COMPOUNDS CONTENT, AND ANTIOXIDANT ACTIVITY OF BASIL}

\author{
Francisco-Javier Yépez-Hernández ${ }^{1}$, Ronald Ferrera-Cerrato ${ }^{1}$, Alejandro Alarcón ${ }^{1 *}$, \\ Julián Delgadillo-Martínez', Ma. Remedios Mendoza-López ${ }^{2}$ y Óscar García-Barradas ${ }^{2}$
}

\begin{abstract}
'Área de Microbiología, Postgrado de Edafología, Colegio de Postgraduados. Km 36.5 Carr. México-Texcoco. 56230, Montecillo, Texcoco de Mora, Estado de México. ${ }^{2}$ Unidad de Servicios de Apoyo en Resolución Analítica (SARA), Universidad Veracruzana. Dr. Luis Castelazo S/N. 91190, Col. Industrial Ánimas, Xalapa, Veracruz, México.
\end{abstract}

*Autor para correspondencia ( aalarconcp@gmail.com)

\section{RESUMEN}

Este trabajo evaluó el efecto de seis concentraciones de nitrógeno $(\mathrm{N})$ $(0,12.5,25,50,75$ y $100 \%)$ en el crecimiento y respuestas fisiológicas de albahaca (Ocimum basilicum L.) cultivada en condiciones de invernadero. Las concentraciones de $\mathrm{N}$ fueron aplicadas con la solución nutritiva Long-Ashton que contiene $160.25 \mu \mathrm{g} \mathrm{N} \mathrm{mL}^{-1}(100 \% \mathrm{~N})$. A los $120 \mathrm{~d}$, se evaluó la altura del tallo (ALT), longitud de raíz (LR), número de hojas (NH), peso seco total (Pstot), área foliar (AF), contenido de compuestos fenólicos solubles totales (CFT), y la actividad antioxidante total (AOX) en hojas. No se observaron diferencias significativas por efecto de la concentración de $\mathrm{N}$ en ALT, LR, NH, Pstot y CFT. La aplicación de $100 \% \mathrm{~N}$ produjo el valor más bajo $(424.8 \mu \mathrm{g}$ $\left.\mathrm{g}^{-1}\right)$ de CFT en comparación con el control $(0 \% \mathrm{~N})$ que obtuvo $826.9 \mu \mathrm{g} \mathrm{g}^{-1}$. AF y AOX presentaron diferencias significativas $(a=0.05)$; las plantas con $75 \% \mathrm{~N}$ mostraron mayor AF $\left(538 \mathrm{~cm}^{2}\right)$ con respecto al control $\left(297 \mathrm{~cm}^{2}\right)$. La mayor AOX se obtuvo en el tratamiento $\sin \mathrm{N}\left(5.9 \mathrm{mM} \mathrm{g}^{-1}\right)$, y la menor con $100 \% \mathrm{~N}\left(3.1 \mathrm{mM} \mathrm{g}^{-1}\right)$. La deficiencia de $\mathrm{N}$ no afectó el crecimiento vegetal, pero aumentó el CFT y la AOX, lo que conviene considerar para mejorar sus propiedades nutraceúticas.

Palabras clave: Ocimum basilicum, planta medicinal, deficiencia de $\mathrm{N}$, fenólicos, antioxidantes.

\section{SUMMARY}

This study evaluated the effects of six nitrogen concentrations $(0,12.5,25$, 50,75 and $100 \%$ ) on growth and physiological responses of basil (Ocimum basilicum L.) under greenhouse conditions. Nitrogen concentrations were supplied via the Long Aston nutrient solution that contains $160.25 \mu \mathrm{g} \mathrm{N}$ $\mathrm{mL}^{-1}$ at $100 \% \mathrm{~N}$. After $120 \mathrm{~d}$, plant height $(\mathrm{H})$, root length $(\mathrm{RL})$, number of leaves (NL), total dry weight (TDW), leaf area (LA), content of total soluble phenolic compounds (TSPC) and total antioxidant activity (TAOX) in leaves were determined. No significant differences were observed on $\mathrm{H}, \mathrm{RL}, \mathrm{NL}$, TDW and TSPC as a response to varied N concentrations. Application of $100 \% \mathrm{~N}$ resulted in lower TSPC $\left(424.8 \mu \mathrm{g} \mathrm{g}^{-1}\right)$ than the control $(0 \% \mathrm{~N})$ with $826.9 \mu \mathrm{g}$ $\mathrm{g}^{-1}$. LA and TAOX showed significant differences $(\mathrm{a}=0.05)$; plants with $75 \% \mathrm{~N}$ had higher LA $\left(538.67 \mathrm{~cm}^{2}\right)$ than the control $\left(296.94 \mathrm{~cm}^{2}\right)$. The greatest TAOX was achieved in control plants $\left(5.9 \mathrm{mM} \mathrm{g}^{-1}\right)$, whereas the lowest value was obtained with $100 \% \mathrm{~N}\left(3.1 \mathrm{mM} \mathrm{g}^{-1}\right)$. N-deficiency did not affect plant growth, but enhanced both TSPC and TAOX. The latter must be taken into account to keep the nutraceutical properties of this plant species.

Index words: Ocimum basilicum, medicinal plants, nitrogen deficiency, phenolics, antioxidants.

\section{INTRODUCCIÓN}

Albahaca (Ocimum basilicum L.) es una planta herbácea representada por al menos 50 variedades que se caracterizan por su aroma originado por compuestos volátiles (Carovic-Stanko et al., 2011; Duke, 1987; Sánchez-Verdugo y Lucero-Flores, 2012). La albahaca se emplea principalmente como un producto culinario, pero además tiene propiedades medicinales (Briskin, 2000). Los compuestos activos de esta planta tienen propiedades insecticidas, nematicidas, fungicidas y antimicrobianas; así mismo, se usa como base de fragancias, champús, lociones, licores y aperitivos (Mendiola-Ubillos y Martín-Montalbán, 2009; Smirnova et al., 2012). Estas propiedades hacen de la albahaca un cultivo con alta demanda; por ello la producen países como Francia, Hungría, Egipto, entre otros, y destacan la Unión Europea, Canadá y Estados Unidos como los principales exportadores (Martínez-Gordillo et al., 2013; Sánchez-Verdugo y Lucero-Flores, 2012).

Las plantas medicinales producen compuestos activos que provocan alguna actividad farmacológica en los organismos vivos, entre los que se incluyen los terpenos, alcaloides y compuestos fenólicos que poseen propiedades antioxidantes (Kwee y Niemeyer, 2011; Muñoz, 2002). Los principales ácidos fenólicos que contiene la albahaca son rosmarínico, chicórico, caftárico y cafeico (Kwee y Niemeyer, 2011; Lee y Scagel, 2009; Toussaint et al., 2007), cuyos contenidos en el tejido vegetal pueden variar por muchos factores, y algunos están involucrados como respuesta ante factores de estrés tanto bióticos como abióticos (Scagel y Lee, 2012). La principal ruta metabólica para la síntesis de compuestos fenólicos en plantas es la del ácido shikímico, la cual inicia con la fenilalanina (Taiz y Zeiger, 2002).

Los ácidos rosmarínico, chicórico y en menor concentración 
caftárico y cafeico, son utilizados en diferentes tratamientos para mejorar la salud humana, para prevenir la obesidad, enfermedades cardiacas, cáncer de colon, desórdenes gastrointestinales, y también para reducir la diabetes (Ignat et al., 2011).

El ácido rosmarínico se utiliza como agente anti-inflamatorio debido a que presenta características analgésicas y antipiréticas, y en el tratamiento de la artritis y lesiones de músculos, ligamentos y tendones para evitar el dolor y la inflamación (Kim et al., 2010). El ácido chicórico es inhibidor de la integrasa del $\mathrm{VIH}-\mathrm{I}$, y ayuda en la moderación del estrés crónico y la depresión (Lee y Scagel, 2009). Además, los compuestos fenólicos totales (CFT) tienen propiedades antioxidantes que son parte importante de los procesos no enzimáticos de defensa contra el estrés oxidativo causado por la formación de especies reactivas de oxígeno (Gill y Tuteja, 2010; Ignat et al., 2011 ; Zulfugarov et al., 2011).

Los CFT han sido muy investigados en los últimos años debido al poder antioxidante que presentan (Sroka y Cisowski, 2003), por su habilidad de disminuir el estrés oxidativo mediante la donación de electrones y átomos de hidrógeno, y de quelatar iones como el hierro y cobre (Agati et al., 2012). Algunos de estos compuestos poseen propiedades terapéuticas y preventivas de ciertas enfermedades crónico-degenerativas, por lo que se les ha acuñado la denominación de nutracéuticos o alimentos funcionales (Berdowska et al., 2013; Briskin, 2000; Impa et al., 2012).

Dentro del área farmacéutica, uno de los objetivos ha sido mejorar el rendimiento de los principios activos presentes en las plantas. Además de usar técnicas moleculares, se ha optado por metodologías más económicas debido a la alta demanda de sus compuestos benéficos (Nascimento y Fett-Neto, 2010). Con el fin de aumentar la concentración de los compuestos fenólicos, las plantas medicinales han sido expuestas a factores bióticos como la infección de microorganismos (Asensio et al., 2012), y factores abióticos como el déficit hídrico, alcalinidad, alta radiación, deficiencia nutrimental, alta luminosidad y humedad de suelos o del sustrato (De la Rosa et al., 2011; Moreno-Rodríguez et al., 2014; Rajashekar et al., 2012).

La fertilización nitrogenada se utiliza para mantener e incrementar los rendimientos de un cultivo. En el caso de plantas medicinales, al igual que en todas las plantas cultivadas, es necesario conocer los niveles óptimos de fertilización que permitan proveer suficiente $\mathrm{N}$ a la planta para maximizar su crecimiento y rendimiento de compuestos funcionales benéficos para la salud humana (Cherry et al., 2008; Ju et al., 2009; Robertson y Vitousek, 2009). Por lo anterior, el presente estudio tuvo como objetivo evaluar el efecto de diferentes concentraciones de nitrógeno aplicadas con solución nutritiva en el crecimiento y fisiología de plantas de albahaca desarrolladas en condiciones de invernadero.

\section{MATERIALES Y MÉTODOS}

\section{Material vegetal}

Se emplearon semillas comerciales de albahaca variedad Hoja Grande (Rancho Los Molinos ${ }^{\circledR}$ ), las cuales fueron desinfectadas con una solución de $\mathrm{NaClO}$ a $2 \% \mathrm{v} / \mathrm{v}$ durante 3 min y se enjuagaron tres veces con agua destilada estéril, para eliminar posibles patógenos presentes en la superficie de la semilla. La germinación de las semillas se hizo en papel filtro húmedo en cajas de Petri a $25^{\circ} \mathrm{C}$ durante $72 \mathrm{~h}$. Las plántulas obtenidas se trasplantaron en macetas de plástico (dos por maceta) que contenían 500 $\mathrm{g}$ de sustrato compuesto de una mezcla de turba-perlitavermiculita $(1: 1: 1, \mathrm{p} / \mathrm{p})$ esterilizada a $121^{\circ} \mathrm{C}$ por $3 \mathrm{~h}$ durante $3 d$.

Las plantas se regaron con solución nutritiva Long-Ashton completa durante las primeras cuatro semanas (Hewitt, 1966), cuya composición general en ( $\left.\mathrm{g} \mathrm{L}^{-1}\right)$ es: $80.8 \mathrm{KNO}_{3}$; $73.6 \mathrm{MgSO}_{4} \cdot 7 \mathrm{H}_{2} \mathrm{O} ; 188.8 \mathrm{Ca}\left(\mathrm{NO}_{3}\right)_{2} \cdot 4 \mathrm{H}_{2} \mathrm{O} ; 36.8 \mathrm{NaH}_{2} \mathrm{PO}_{4} \cdot \mathrm{H}_{2} \mathrm{O}$; $1.69 \mathrm{MnSO} 4 \cdot \mathrm{H}_{2} \mathrm{O} ; 0.25 \mathrm{CuSO}_{4} \cdot 5 \mathrm{H}_{2} \mathrm{O} ; 0.29 \mathrm{ZnSO}_{4} \cdot 7 \mathrm{H}_{2} \mathrm{O} ; 3.10$ $\mathrm{H}_{3} \mathrm{BO}_{3} ; 5.90 \mathrm{NaCl} ; 0.088\left(\mathrm{NH}_{4}\right)_{6} \mathrm{MO}_{7} \mathrm{O}_{24} \cdot 4 \mathrm{H}_{2} \mathrm{O} ; 4.9 \mathrm{FeC}_{6} \mathrm{H}_{5} \mathrm{O}_{7} ;$ y $4.9 \mathrm{H}_{3} \mathrm{C}_{6} \mathrm{H}_{5} \mathrm{O}_{7} \cdot \mathrm{H}_{2} \mathrm{O}$. Este experimento se llevó a cabo en condiciones de invernadero del 10 de agosto de 2012 al 23 de diciembre del 2012, a una temperatura promedio de $20.4{ }^{\circ} \mathrm{C}$ y humedad relativa promedio de $34.4 \%$, según registros obtenidos con un dispositivo $\mathrm{HOBO}{ }^{\circledR} \mathrm{TEMP} / \mathrm{RH}$ 1000 Series (Spectrum Technologies, USA).

\section{Aplicación de fertilización nitrogenada}

A partir de la cuarta semana después del trasplante y hasta la cosecha (4 meses), las plantas se regaron con la solución nutritiva Long-Ashton (Hewitt, 1966) preparada con diferentes dosis de nitrógeno. La solución testigo tuvo una concentración óptima de $160.25 \mu \mathrm{g}$ de $\mathrm{N} \mathrm{mL}^{-1}$ (100\% $\mathrm{N})$, y a partir de ésta se prepararon las diferentes diluciones: $75,50,25,12.5$ y $0 \%$ N. Cada tratamiento consistió de cinco repeticiones $(n=5)$ que fueron distribuidas en un diseño experimental completamente al azar.

\section{Cosecha, evaluación del crecimiento y análisis nutrimental}

Cuatro meses (120 d) después de la aplicación de los tratamientos de nitrógeno, se evaluó el crecimiento de las cinco plantas en cada tratamiento, con las variables altura del tallo $(\mathrm{cm})$, longitud de raíz $(\mathrm{cm})$, número de hojas, 
pesos secos de tallo, hojas, raíz y total. El área foliar se determinó con un medidor de área foliar (LICOR LI-3100®, USA). Además, en el tejido foliar se cuantificó el nitrógeno total por el método semi-microKjeldahl (Bremner, 1965) y el fósforo total (Hanson, 1950), los cuales se expresaron en porcentaje y como contenido total en follaje.

\section{Determinación de compuestos fenólicos solubles totales y actividad antioxidante total en hojas}

La determinación de los compuestos fenólicos solubles totales (CFT) se llevó a cabo por el método de Folin-Ciocalteau (Folin y Ciocalteau, 1927; Lester et al., 2012; Singleton y Rossi, 1965) y la actividad antioxidante total (AOX) por el método de reducción de DPPH (Brand-Williams et al., 1995). Para la extracción, de cada planta por tratamiento $(\mathrm{n}=5)$, se pesaron de 0.150 a $0.200 \mathrm{~g}$ de tejido foliar macerado con $3 \mathrm{~mL}$ de metanol a $80 \%$. El extracto se centrifugó a 19,940 x g por $15 \mathrm{~min}$, y las muestras se almacenaron en refrigeración a $4^{\circ} \mathrm{C}$ hasta su análisis.

Para la determinación de los CFT se tomaron alícuotas de $30 \mu \mathrm{L}$ de los extractos y se colocaron por duplicado en microplacas de 96 pozos. A continuación, se aplicaron 150 $\mu \mathrm{L}$ del reactivo de Folin-Ciocalteau y $90 \mu \mathrm{L}$ de $\mathrm{Na}_{2} \mathrm{CO}_{3} 1$ $\mathrm{N}$. El volumen final por pozo fue de $270 \mu \mathrm{L}$. Posteriormente, se procedió a incubar la placa por 30 min y se leyó la absorbancia a $725 \mathrm{~nm}$. Los resultados se expresaron en $\mu \mathrm{g}$ de ácido clorogénico por gramo tejido fresco $\left(\mu \mathrm{g} \mathrm{g}^{-1}\right)$. La determinación de la actividad antioxidante (AOX) se realizó por el método de reducción de DPPH (1,1-difenil-2-picrilhidrazilo), con $15 \mu \mathrm{L}$ de los extractos obtenidos colocados en microplacas de 96 pozos por duplicado, a los que se adicionaron $270 \mu \mathrm{L}$ de una solución de DPPH, y se tomó la absorbancia inicial a $515 \mathrm{~nm}$; después de $15 \mathrm{~min}$ de incubación a temperatura ambiente, se tomó nuevamente la absorbancia a $515 \mathrm{~nm}$. Los resultados se expresaron en $\mathrm{mM}$ de Trolox por gramo de tejido fresco $\left(\mathrm{mM} \mathrm{g}^{-1}\right)$.

\section{Análisis estadísticos}

Los datos obtenidos del experimento fueron sometidos a un análisis de varianza y a una prueba de comparación de medias de t de Student $(a=0.05)$ mediante el programa SAS para Windows (SAS Institute, 2002).

\section{RESULTADOS}

\section{Crecimiento y estado nutrimental}

La fertilización nitrogenada no causó diferencias significativas en la altura del tallo, longitud de raíz, ni en el peso seco de las plantas (Cuadros 1 y 2). En contraste, el número de hojas aumentó significativamente $(P \leq 0.001)$ conforme la dosis de nitrógeno incrementó; el tratamiento con $100 \%$ N registró mayor número de hojas (49.6) por planta, mientras que el tratamiento con $0 \% \mathrm{~N}$ presentó menor número con 25 hojas (Cuadro 1). De manera similar, el área foliar presentó diferencias significativas $(P \leq 0.001)$ por efecto de la dosis de nitrógeno; el tratamiento con $75 \%$ $\mathrm{N}$ presentó el valor más alto $\left(453.9 \mathrm{~cm}^{3}\right)$, mientras que los tratamientos con $0,12.5$ y $25 \% \mathrm{~N}$ obtuvieron los valores más bajos. El tratamiento con $75 \% \mathrm{~N}$ mostró $44.7 \%$ más área foliar que el tratamiento con $0 \% \mathrm{~N}$.

El porciento de nitrógeno total y el contenido total de nitrógeno foliar presentaron diferencias significativas entre tratamientos ( $P \leq 0.001)$. Los tratamientos con 50, 75 y $100 \%$ de $\mathrm{N}$ presentaron los valores más elevados de nitrógeno total en comparación con el tratamiento de 12.5 $\%$ de $\mathrm{N}$ que presentó el promedio más bajo (Figura 1A). Así mismo, el tratamiento con $75 \%$ de $\mathrm{N}$ presentó el mayor contenido de nitrógeno foliar con $0.033 \mathrm{mg} \mathrm{g}^{-1}$, mientras que los tratamientos con 0, 12.5 y $25 \%$ de $\mathrm{N}$ presentaron los valores más bajos, y el tratamiento con $12.5 \%$ de $\mathrm{N}$ mostró el valor más bajo con $0.016 \mathrm{mg} \mathrm{g}^{-1}$ (Figura 1B).

El porcentaje y contenido total de P-foliar presentaron

Cuadro 1. Altura de planta, longitud de raíz, número de hojas y área foliar en albahaca (Ocimum basilicum L.), medidas 120 d después de aplicar seis diferentes concentraciones de nitrógeno.

\begin{tabular}{|c|c|c|c|c|}
\hline \multirow{2}{*}{ Tratamiento (\% N) } & Altura & Longitud de raíz & \multirow{2}{*}{ Número de hojas } & \multirow{2}{*}{ Área foliar $\left(\mathrm{cm}^{3}\right)$} \\
\hline & \multicolumn{2}{|c|}{$(\mathrm{cm})$} & & \\
\hline 0 & $22.7 \pm 7.9 a$ & $19.6 \pm 0.5 a$ & $25 \pm 5.5 b$ & $335.8 \pm 83.1 b$ \\
\hline 12.5 & $36.0 \pm 6.0 \mathrm{a}$ & $20.0 \pm 0.9 a$ & $36.7 \pm 4.4 \mathrm{ab}$ & $365.9 \pm 25.1 b$ \\
\hline 25 & $29.2 \pm 7.1 \mathrm{a}$ & $16.6 \pm 1.7 \mathrm{a}$ & $36 \pm 3.5 \mathrm{ab}$ & $344.9 \pm 73.2 b$ \\
\hline 50 & $29.3 \pm 4.6 a$ & $19.9 \pm 2.5 a$ & $38.7 \pm 6.4 \mathrm{ab}$ & $440.9 \pm 39.1 \mathrm{ab}$ \\
\hline 75 & $26.4 \pm 3.4 \mathrm{a}$ & $20.2 \pm 0.9 a$ & $34.7 \pm 11.7 \mathrm{ab}$ & $606.9 \pm 89.9 a$ \\
\hline 100 & $27.9 \pm 7.4 a$ & $22.3 \pm 3.1 \mathrm{a}$ & $49.7 \pm 5.0 \mathrm{a}$ & $453.9 \pm 44.6 \mathrm{ab}$ \\
\hline
\end{tabular}

Medias \pm error estándar $(n=5)$. Valores con letras idénticas no son estadísticamente diferentes entre tratamientos ( $t$ de Student, $a=0.05)$. 
Cuadro 2. Peso seco de tallo, hojas, raíz y total en albahaca (Ocimum basilicum L.), a 120 d después de aplicar seis diferentes concentraciones de nitrógeno.

\begin{tabular}{lcccc}
\hline \multirow{2}{*}{ Tratamiento } & Peso seco total & Peso seco de tallo & Peso seco de hojas & Peso seco de raíz \\
\cline { 2 - 5 } & \multicolumn{5}{c}{$(\mathrm{g})$} \\
\hline 0 & $1.2 \pm 0.3 \mathrm{a}$ & $0.3 \pm 0.1 \mathrm{a}$ & $0.5 \pm 0.1 \mathrm{a}$ & $0.5 \pm 0.2 \mathrm{a}$ \\
12.5 & $1.5 \pm 0.03 \mathrm{a}$ & $0.4 \pm 0.04 \mathrm{a}$ & $0.5 \pm 0.01 \mathrm{a}$ & $0.5 \pm 0.03 \mathrm{a}$ \\
25 & $1.4 \pm 0.5 \mathrm{a}$ & $0.3 \pm 0.1 \mathrm{a}$ & $0.5 \pm 0.1 \mathrm{a}$ & $0.6 \pm 0.3 \mathrm{a}$ \\
50 & $1.3 \pm 0.1 \mathrm{a}$ & $0.3 \pm 0.08 \mathrm{a}$ & $0.5 \pm 0.04 \mathrm{a}$ & $0.4 \pm 0.02 \mathrm{a}$ \\
75 & $1.7 \pm 0.4 \mathrm{a}$ & $0.4 \pm 0.13 \mathrm{a}$ & $0.7 \pm 0.1 \mathrm{a}$ & $0.5 \pm 0.2 \mathrm{a}$ \\
100 & $1.3 \pm 0.2 \mathrm{a}$ & $0.3 \pm 0.05 \mathrm{a}$ & $0.6 \pm 0.1 \mathrm{a}$ & $0.4 \pm 0.1 \mathrm{a}$ \\
\hline
\end{tabular}

Medias \pm error estándar $(n=5)$. Valores con letras idénticas no son estadísticamente diferentes entre tratamientos $(t$ de Student, $a=0.05)$.

diferencias significativas entre tratamientos $(P \leq 0.001)$. Los tratamientos con la aplicación de 100, 75, 50 y $25 \%$ de $\mathrm{N}$ presentaron los valores más altos de $\mathrm{P}$ con $0.77 \%$ en promedio (Figura 1C). El tratamiento con 0 y $12.5 \%$ de N mostraron los valores más bajos de $\mathrm{P}$ con una media de $0.58 \%$. El contenido total de P-foliar fue mayor en el tratamiento con $75 \%$, en tanto que los tratamientos con 12.5 y $0 \%$ de $\mathrm{N}$ presentaron los valores más bajos (Figura 1D).

\section{Compuestos fenólicos y actividad antioxidante total en albahaca}

Los CFT en hojas tendió a incrementar conforme la concentración de $\mathrm{N}$ disminuyó en la solución nutritiva; sin embargo, las diferencias entre los tratamientos no fueron significativas de acuerdo al análisis estadístico. El valor más alto se obtuvo en el tratamiento de $0 \%$ de $\mathrm{N}$ con 826.93 $\mu \mathrm{g} \mathrm{g}^{-1}$, mientras que con la aplicación de 75 y $100 \%$ de $\mathrm{N}$ el CFT fue de $426.7 \mu \mathrm{g} \mathrm{g}^{-1}$, en promedio (Figura 1E). La AOX disminuyó significativamente conforme incrementó la concentración de $N(P \leq 0.001)$. La mayor actividad antioxidante se reportó en los tratamientos 0 y $12.5 \%$ N (5.94 y $5.37 \mathrm{mM} \mathrm{g}^{-1}$, respectivamente), mientras que los tratamientos con 100 y $75 \%$ de $N$ mostraron la menor actividad $\left(2.6 \mathrm{mM} \mathrm{g}^{-1}\right)$ (Figura 1F).

\section{DISCUSIÓN}

\section{Crecimiento y estado nutrimental}

El nitrógeno es fundamental en las plantas para la síntesis de ácidos nucleicos, proteínas, porfirinas de la clorofila y algunos citocromos necesarios para la fotosíntesis y la respiración (Alcántar-González y Trejo-Téllez, 2009; Azcón-Bieto y Talón, 2008; Salisbury y Ross, 1994). Los niveles óptimos de nitrógeno tienden a promover una rápida división y elongación celular, por lo cual era de esperarse que el crecimiento de albahaca resultara limitado por una baja disponibilidad de nitrógeno (Alcántar-González y
Trejo-Téllez, 2009; Giorgio et al. 2009).

Matsumoto et al. (2013) y Frabboni et al. (2011) reportaron que la aplicación de una mayor dosis de $\mathrm{N}$ resultó en incrementos en la biomasa y altura de $O$. basilicum crecida en condiciones de campo, aunque los efectos benéficos de la aplicación de $\mathrm{N}$ fueron reducidos por altas dosis de potasio. Lo anterior resalta la interacción del $\mathrm{N}$ y el $\mathrm{K}$ en el crecimiento y desarrollo vegetal, aunque en este estudio no se contempló esta posible interacción nutrimental. La ausencia de diferencias en el crecimiento vegetal y biomasa generada por efecto de distintas dosis de nitrógeno en albahaca, sugiere que el desarrollo de esta especie vegetal es independiente a la cantidad de nitrógeno aplicado, como se ha discutido por Martínez-Carrasco et al. (2005) y (Millard, 2006).

González-García et al. (2009) observaron que la albahaca incrementa su altura en cultivo hidropónico con aplicación de solución nutritiva con una relación de amonio/nitrato 20:80. En contraste, Sifola y Barbieri (2006) demostraron que la fertilización de tres cultivos de albahaca crecidos en campo con distintas concentraciones de nitrógeno $(0$, 100 y $300 \mathrm{~kg} \mathrm{~N} \mathrm{ha}^{-1}$ ) no produjo cambios significativos en la altura. Así mismo, Carrasco et al. (2007) indicaron que la altura de albahaca no presentó diferencias significativas por efecto de la conductividad eléctrica de la solución nutritiva. En el presente experimento las soluciones nutritivas aplicadas registraron $\mathrm{pH}$ de 5.5 a 5.6 y CE promedio de $52.1 \mu \mathrm{cm}^{-1}$, por lo que no fueron factores que hayan afectado el crecimiento. Lo anterior permite inferir que la altura de albahaca es independiente a la cantidad de nitrógeno aplicado.

La longitud de raíz mostró valores semejantes entre tratamientos, lo cual indica que el alargamiento del sistema radical también es independiente de las dosis de nitrógeno. Es importante recalcar que existieron fuentes alternas de nitrógeno, ya que la turba del sustrato utilizado puede contener 

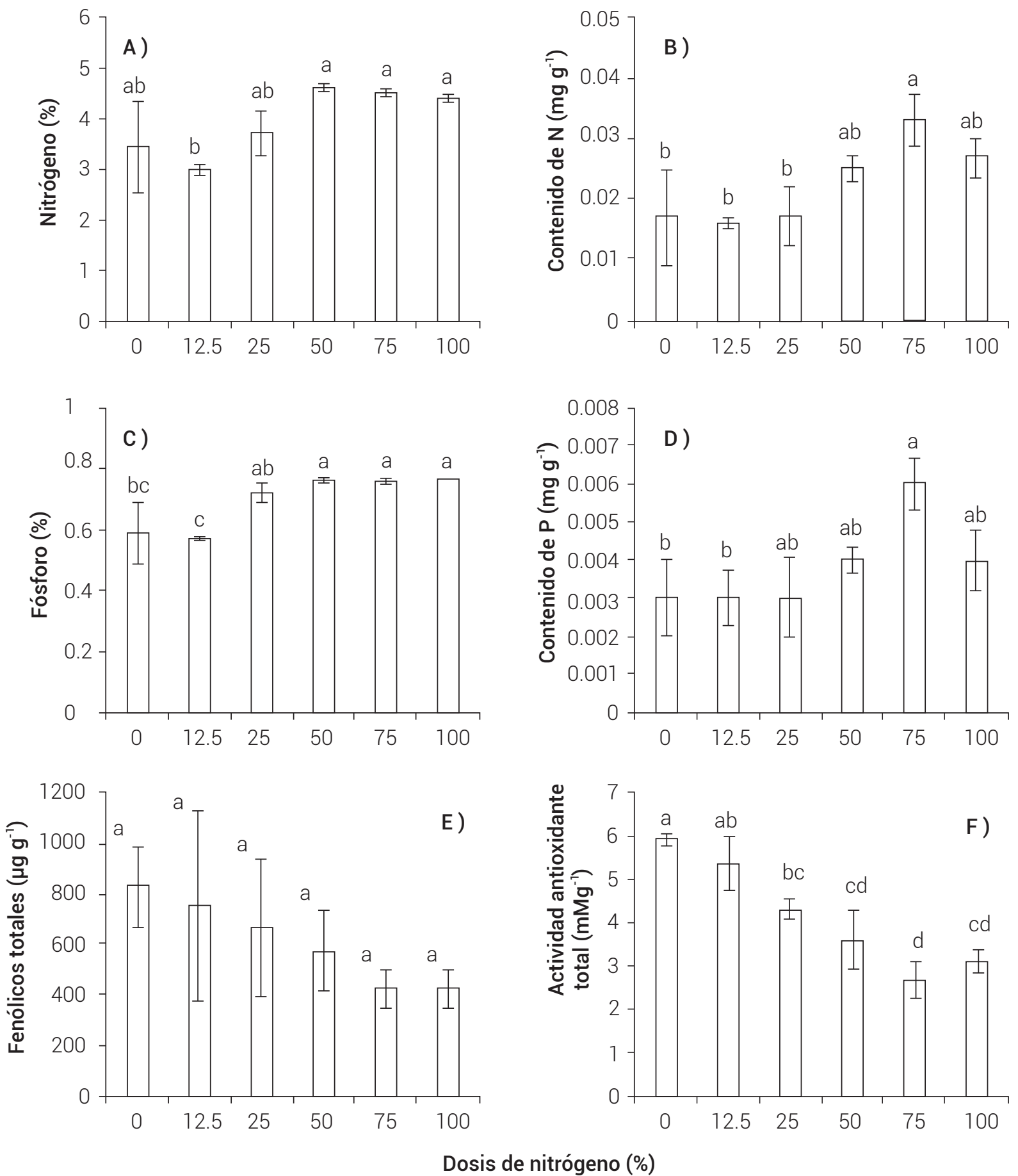

Figura 1. Contenidos de nitrógeno, fósforo, compuestos fenólicos solubles totales, y actividad antioxidante total en tejido foliar de albahaca (Ocimum basilicum L.), a 120 d después de aplicar seis concentraciones de nitrógeno. Medias \pm error estándar $(n=5)$. Letras idénticas sobre las barras son estadísticamente iguales (t de Student, $a=0.05)$. 
cerca de $1 \%$ de N (Resh 2006); dicho N pudo haber sido tomado por la planta en los tratamientos con limitada fertilización nitrogenada, como sugirieron Dayegamiye e Isfan (1991). No se encontraron reportes para albahaca acerca del desarrollo radical bajo estrés de nitrógeno para fortalecer esta discusión. Sin embargo, algunos reportes indican que la aplicación de nitrógeno no afectó la longitud de la raíz en trigo (Triticum aestivum) o berenjena (Solanum melongena) (Rasmussen et al., 2015; Zhang et al., 2014).

El peso seco de las plantas (raíz, tallo, hojas y total) no mostró diferencias significativas por efecto de las dosis de nitrógeno. Estos datos son similares a los reportados por Tarchoune et al. (2013), al demostrar que el peso seco total de hojas en albahaca fue de $1.2 \mathrm{~g}$ en etapas juveniles con aplicación de solución de Hoagland diluida ocho veces. Así mismo, se ha demostrado que el peso seco total foliar a los 30 d tenía una media aproximada entre 3.8 a 3.9 g (Tarchoune et al., 2010; Tarchoune et al., 2012); resultados que son superiores a los obtenidos en la presente investigación (1.2 a $1.7 \mathrm{~g})$ a los $120 \mathrm{~d}$. Los datos reportados por Tarchoune et al. (2010), Tarchoune et al. (2012) y Tarchoune et al. (2013) fueron obtenidos en cultivos hidropónicos con una solución nutritiva rica en nitrógeno, lo que favoreció el desarrollo vegetal. Por otro lado, cuando una planta crece en maceta, su crecimiento siempre está limitado por el tamaño del recipiente. Es probable que en esta investigación, la maceta empleada haya limitado el desarrollo de la raíz, lo que pudo originar una biomasa radical similar entre tratamientos.

La aplicación de 100 \% N produjo mayor número de hojas y las plantas con $75 \% \mathrm{~N}$ presentaron mayor área foliar con respecto a los tratamientos con 0, 12.5 y $25 \% \mathrm{~N}$. Tarchoune et al. (2010) mostraron que la albahaca registra en promedio 90 hojas y $1893 \mathrm{~cm}^{2}$ de área foliar durante su desarrollo en cultivo hidropónico. Por su parte, Sifola y Barbieri (2006) mostraron que al incrementar la dosis de nitrógeno, el número de hojas de albahaca también aumentó. La presente investigación demuestra que el área foliar y el número de hojas incrementaron al aplicar mayor concentración de nitrógeno.

Sifola y Barbieri (2006) reportaron que no existen diferencias significativas en el contenido de nitrógeno foliar al fertilizar un cultivo de albahaca en condiciones de campo con 0 y $100 \mathrm{~kg} \mathrm{~N} \mathrm{ha}^{-1}$; sin embargo, la aplicación de 300 $\mathrm{kg} \mathrm{N} \mathrm{ha-1}$ aumentó el contenido de nitrógeno foliar. Los resultados de estos autores son similares a los encontrados en la presente investigación, donde la acumulación de nitrógeno fue mayor al aplicar mayor cantidad de este nutrimento.

\section{Compuestos fenólicos y actividad antioxidante total en albahaca}

Kwee y Niemeyer (2011) indican que los CFT son dependientes de la variedad de albahaca; por ejemplo, la variedad Spice tiene mayor contenido de estos compuestos (17.6 $\left.\mathrm{mg} \mathrm{g}^{-1}\right)$ en comparación con la variedad Sweet Dani Lemon (3.5 $\mathrm{mg} \mathrm{g}^{-1}$ ). Por su parte, Scagel y Lee (2012) reportaron que las variedades Cinnamon, Sweet Dani, Siam Queen y Red Rubin en albahaca poseen concentraciones de estos compuestos de 7.1, 5.6, 8.0 y $5.9 \mathrm{mg} \mathrm{g}^{-1}$, respectivamente.

Los resultados obtenidos en el presente trabajo (826.9 $\mu \mathrm{g} \mathrm{g}^{-1}$, valor máximo) están por debajo de los valores reportados por los autores arriba mencionados. Al respecto, es conveniente considerar que el análisis de CFT por el método de Folin-Ciocalteau sufre de interferencia por sustancias como el ácido ascórbico, ácidos orgánicos, azúcares, aminas aromáticas, dióxido de azufre, ácidos orgánicos, hierro, además de los flavonoides, las antocianinas y los taninos (Prior et al. 2005; Ignat et al., 2011). Sin embargo, la mejor explicación es que las concentraciones de estos compuestos dependen en alto grado de las condiciones ambientales y de la etapa fenológica de la planta. Las evaluaciones hechas para este experimento se realizaron antes de la floración, cuando la planta estaba madura. Según Witzell et al. (2003), conforme una planta madura su reserva de compuestos fenólicos disminuye, como consecuencia de su incorporación en la pared celular del tallo en forma de lignina.

La aplicación de concentraciones de $\mathrm{N}$ mayores a 0.1 mM o 0.5 mM, resultó en menor contenido de compuestos fenólicos (ácido rosmarínico y cafeico) y menor actividad antioxidante, respectivamente, en dos cultivares de $\mathrm{O}$. basilicum (Nguyen y Neimeyer, 2008). Además, Nguyen et al. (2010) reportaron que la AOX y el CFT de tres variedades de albahaca disminuyeron con la fertilización de $1.0 \mathrm{mM}$ de K, en comparación con la aplicación de 2.0 mM de K, en magnitudes que dependieron de la variedad; este efecto también ha sido discutido por Kwee y Niemeyer (2011). Lo anterior indica que los valores determinados en el presente estudio son similares a los reportados en otras investigaciones; y de manera específica, la aplicación de $100 \%$ de $\mathrm{N}$ resultó en menor actividad antioxidante $\left(3.12 \mathrm{mM} \mathrm{g}^{-1}\right)$.

La AOX aquí determinada considera la actividad de compuestos enzimáticos y no enzimáticos extraídos del tejido foliar. El estudio de los compuestos enzimáticos o no enzimáticos con actividad antioxidante en plantas aromáticas o medicinales ha cobrado importancia por sus propiedades farmacéuticas, ya que contribuyen a reducir daños causado por radicales libres (Berdowska et al., 2013; Carol 
y Dolan, 2006; Foyer y Noctor, 2009; Garg y Manchanda, 2009; Gulçin et al., 2007; Impa et al., 2012; Sergent et al., 2012; Sharma y Bhat, 2009; Sharma et al., 2012).

En general, la aplicación de diversas dosis de nitrógeno en la solución nutritiva de Long Asthon, no afectó el crecimiento, desarrollo y nutrición de la planta de albahaca. Sin embargo, las dosis mayores a $25 \% \mathrm{~N}$ disminuyeron el CFT y la AOX de esta especie vegetal. Lo anterior podría considerarse en sistemas de producción de 0 . basilicum, para favorecer sus propiedades nutraceúticas.

\section{CONCLUSIONES}

La fertilización nitrogenada no afectó el crecimiento de albahaca, pero alteró negativamente al contenido de compuestos fenólicos totales (CFT) y a la actividad antioxidante (AOX). La aplicación de dosis mayores a $25 \%$ N disminuyó la acumulación de CFT y la AOX, de modo que para producir mayores cantidades de CFT y de AOX en plantas de albahaca, se propone usar dosis bajas de nitrógeno.

\section{Agradecimientos}

Esta investigación formó parte del proyecto SEP-CONACYT-130262. FJYH agradece el apoyo a CONACyT durante sus estudios de maestría. Los autores agradecen la revisión y comentarios de dos revisores anónimos que fortalecieron el presente manuscrito.

\section{BIBLIOGRAFÍA}

Agati G., E. Azzarello, S. Pollastri and M. Tattini (2012) Flavonoids as antioxidants in plants: Location and functional significance. Plant Science 196:67-76.

Alcántar-González G. y L. I. Trejo-Téllez (eds.) (2009) Nutrición de Cultivos. Ed. Mundi-Prensa, México, D.F. 454 p.

Asensio D., F. Rapparini and J. Peñuelas (2012) AM fungi root colonization increases the production of essential isoprenoids vs. nonessential isoprenoids especially under drought stress conditions or after jasmonic acid application. Phytochemistry 77:149-161.

Azcon-Bieto J. y M. Talón (2008) Fundamentos de Fisiología Vegetal. Ed. Interamericana-McGraw-Hill, Madrid. 522 p.

Berdowska I., B. Zielinski, I. Fecka, J. Kulbacka, J. Saczko and A. Gamian (2013) Cytotoxic impact on phenolics from Lamiaceae species on human breast cancer cells. Food Chemistry 141:1313-1321.

Brand-Williams W, M. E. Cuvelier and C. Berset (1995) Use of a free radical method to evaluate antioxidant activity. Food Science and Technology 28:25-30

Bremner J. M. (1965) Inorganic forms of nitrogen. In: Methods of Soil Analysis. Part 2. C. A. Black et al. (eds.). Agronomic Monographies 9, ASA, Madison, WI. pp:643-698.

Briskin D. P. (2000) Medicinal plants and phytomedicines. Linking plant biochemistry and physiology to human health. Plant Physiology 124:507-514.

Carol R. J. and L Dolan (2006) The role of reactive oxygen species in cell growth: lessons from root hairs. Journal of Experimental Botany 57:1829-1834

Carovic-Stanko K., Z. Liber, O. Politeo, F. Strikic, I. Kolak, M. Milos and Z. Satovic (2011) Molecular and chemical characterization of the most widespread Ocimum species. Plant Systematics and Evolution 294:253-262.
Carrasco G., P. Ramírez and H. Vogel (2007) Effect of the electrical conductivity of the nutrient solution on yield and essential oil in basil grown by NFT. Idesia 25:59-62.

Cherry K. A., M. Shepherd, P. J. A. Withers and S. J. Mooney (2008) Assessing the effectiveness of actions to mitigate nutrient loss from agriculture: A review of methods. Science of the Total Environment 406:1-23.

Dayegamiye A. N. and D. Isfan (1991) Chemical and biological changes in compost of wood shavings, sawdust and peat moss. Canadian Journal of Soil Science 71:475-484.

De la Rosa M. C. L., R. Ferrera-Cerrato, A. Alarcón, M. J. Sánchez-Colin and O. D. Muñoz-Muñiz (2011) Arbuscular mycorrhizal fungi and potassium bicarbonate enhance the foliar content of the vinblastine alkaloid in Catharanthus roseus. Plant Soil 349:367-376.

Duke J. A. (1987) Handbook of Medicinal Herbs. CRC Press Inc. Florida, USA. $896 \mathrm{p}$.

Folin 0. and V. Ciocalteau (1927) On tyrosine and triptophans determinations in proteins. The Journal of Biological Chemistry 73:627250.

Foyer C.H. and G. Noctor (2009) Redox regulation in photosynthetic organisms: signaling, acclimation, and practical implications. Antioxidants \& Redox Signaling 11:861-905.

Frabboni L., G. de Simone and V. Russo (2011) The influence of different nitrogen treatments on the growth and yield of basil (Ocimum basilicum L.) Journal of Chemistry and Chemistry Engineering 5:799-803.

Garg N. and G. Manchanda (2009) ROS generation in plants: Boon or Bane? Plant Biosystems 143:81-96.

Gill S. S. and N. Tuteja (2010) Reactive oxygen species and antioxidant machinery in abiotic stress tolerance in crop plants. Plant Physiology and Biochemistry 48:909-930.

Giorgio A., M. Mingozzi, M. Madeo, G. Speranza and M. Coccuci (2009) Effect of nitrogen starvation on the phenolic metabolism and antioxidant properties of yarrow (Achillea collina Becker ex Rchb.) Food Chemistry 114:204-211.

González-García J. L., M. N. Rodríguez-Mendoza, P. Sánchez-García and E. A. Gaytán-Acuña (2009) Ammonium/Nitrate ratio in the production of aromatic herbs in hydroponics. Agricultura Técnica en México 35:5-11.

Gulçin I., M. Elmastas and H. Y. Aboul-Enein (2007) Determination of antioxidant and radical scavenging activity of basil (Ocimum basilicum L. Family Lamiaceae) assayed by different methodologies. Phytotherapy Research 21:354-361.

Hanson W. C. (1950) The photometric determination of phosphorus in fertilizers using the phosphovanado-molybdate complex. Science of Food and Agriculture 1:172-173.

Hewitt E. J. (1966) The composition of nutrient solution. In: Sand and Water Culture Methods Used in the Study of Plant Nutrition. E. J. Hewitt (ed.). Commonwealth Agricultural Bureau, United Kingdom. pp:187-246.

Ignat I., I. Volf and V. I. Popa (2011) A critical review of methods for characterization of polyphenolic compounds in fruits and vegetables. Food Chemistry 126:1821-1835.

Impa S.M., S. Nadaradjan and S. V. K. Jagadish (2012) Drought stress induced reactive oxygen species and anti-oxidants in plants. In: Abiotic Stress Responses in Plants. P. Ahmad and M. N. V. Prasad. Metabolism, Productivity and Sustainability. Springer New York, USA. pp:131-147.

Ju X. T., G. X. Xing, X. P. Chen, S. L. Zhang and Z. L. Liu (2009) Reducing environmental risk by improving $\mathrm{N}$ management in intensive Chinese agricultural systems. Proceedings of the National Academy of Sciences of the Unites States of America 106:3041-3046.

Kim H. J., T. H. Kim, K. C. Kang, H. B. Pyo and H. H. Jeong (2010) Microencapsulation of rosmarinic acid using polycaprolactone and various surfactants. International Journal of Cosmetic Science 32:185-191.

Kwee E. M. and E. D. Niemeyer (2011) Variations in phenolic composition and antioxidant properties among 15 basil (Ocimum basilicum L.) cultivars. Food Chemistry 128:1044-1050.

Lee J. and C. F. Scagel (2009) Chicoric acid found in basil (Ocimum basilicum L.) leaves. Food Chemistry 115:650-656.

Lester G. E., K. S. Lewers, M. B. Medina and R. A. Saftner (2012) Comparative analysis of strawberry total phenolics via Fast Blue BB vs. 
Folin-Ciocalteu: Assay interference by ascorbic acid. Journal of Food Composition and Analysis 27:102-107.

Martínez-Carrasco R., P. Pérez and R. Morcuende (2005) Interactive effects of elevated $\mathrm{CO}_{2}$, temperature and nitrogen on photosynthesis of wheat grown under temperature gradient tunnels. Environmental and Experimental Botany 54:49-59.

Martínez-Gordillo M., I. Fragoso-Martínez, M. R. García-Peña, y 0. Montiel (2013) Géneros de Lamiaceae de México, diversidad y endemismo. Revista Mexicana de Biodiversidad 84:30-86.

Matsumoto S. N., G. S. Araujo and A. E. S. Viana (2013) Growth of sweet basil depending on nitrogen and potassium doses. Horticultura Brasileira 31:489-493.

Mendiola-Ubillos M. A. y J. Martín-Montalbán (2009) Plantas Aromáticas Gastronómicas. Ed. Mundi-Prensa. México, D. F. 191 p.

Millard P. (2006) The accumulation and storage of nitrogen by herbaceous plants. Plant, Cell \& Environment 11:1-8.

Moreno-Rodríguez A., J. Vázquez-Medrano, L. B. Hernández-Portilla, I Peñalosa-Castro, M. Canales-Martínez, A. Orozco-Segovia, M. JiménezEstrada, L Colville, H. W. Pritchard and C. M. Flores-Ortiz (2014) The effect of light and soil moisture on the accumulation of three flavonoids in the leaves of Mexican oregano (Lippia graveolens Kunth). Journal of Food, Agriculture \& Environment 12:12721279

Muñoz F. (2002) Plantas Medicinales y Aromáticas: Estudio, Cultivo y Procesado. Ed. Mundi-Prensa. México, D. F. 365 p.

Nascimento N. C. and A. G. Fett-Neto (2010) Plant secondary metabolism and challenges in modifying its operation: an overview. In: Plant Secondary Metabolism Engineering, Methods in Molecular Biology. A. G. Fett-Neto (ed.). @ Springer Science+Business Media, LLC 2010643. pp:1-14.

Nguyen P. and E. D. Niemeyer (2008) Effects of nitrogen fertilization on the phenolic composition and antioxidant properties of basil (Ocimum basilicum L.). Journal of Agricultural and Food Chemistry 56:8685-8691.

Nguyen P. M., E. M. Kwee and E. D. Niemeyer (2010) Potassium rate alters the antioxidant capacity and phenolic concentration of basil (Ocimum basilicum L.) leaves. Food Chemistry 123:1235-1241.

Prior R. L., X. Wu and K. Schaich (2005) Standarized methods for the determination of antioxidant capacity and phenolics in food and dietary supplements. Journal of Agriculture and Food Chemistry 53:4290-4302.

Rajashekar C. B., O. Myung-Min and E. E. Carey (2012) Organic crop management enhances chicoric acid content in lettuce. Food and Nutrition Science 3:1296-1302

Rasmussen I. S., D. B. Dresbøll and K. Thorup-Kristensen (2015) Winter wheat cultivars and nitrogen $(\mathrm{N})$ fertilization- Effects on root growth, $\mathrm{N}$ uptake efficiency and $\mathrm{N}$ use efficiency. European Journal of Agronomy 68:38-49.

Resh H. M. (2006) Cultivos Hidropónicos. Ed. Mundi-Prensa. Mexico, D. F. $558 \mathrm{p}$.

Robertson G. P. and P. M. Vitousek (2009) Nitrogen in agriculture: Balancing the cost of an essential resource. Annual Review of Environmental Resources 34:97-125

SAS Institute (2002) The SAS system for windows, ver. 9.0. SAS Institute Inc., Cary, North Carolina, USA.

Salisbury F. B. and C. W. Ross (1994) Plant Physiology. Fourth ed.. Wadsworth Publishing. Belmont, California, USA. 682 p.
Sánchez-Verdugo C. y J. M. Lucero-Flores (2012) Nichos de mercado de especies aromáticas orgánicas tipo gourmet. Centro de Investigaciones Biológicas del Noroeste, S. C. La Paz, Baja California, México. $72 \mathrm{p}$

Scagel C. F. and J. Lee (2012) Phenolic composition of basil plants is differentially altered by plant nutrient status and inoculation with mycorrhizal fungi. HortScience 47:660-671.

Sergent T., J. Vanderstraeten, J. Winand, P. Beguin and Y. J. Schneider (2012) Phenolic compounds and plant extracts as potential natural anti-obesity substances. Food Chemistry 135:68-73.

Sharma O. P. and TK Bhat (2009) DPPH antioxidant assay revisited. Food Chemistry 113:1202-1205.

Sharma P., A. B. Jha, R. S. Dubey and M. Pessarakli (2012) Reactive oxygen species, oxidative damage and antioxidant defense mechanism in plant under stressful conditions. Journal of Botany 2012:1-26

Sifola M. I. and G. Barbieri (2006) Growth, yield and essential oil content of three cultivars of basil grown under different levels of nitrogen in the field. Scientia Horticulturae 108:408-413.

Singleton V. L. and J. A. Rossi (1965) Colorimetry of total phenolics with phosphomolybdic-phosphotungstic acid reagents. American Journal of Enology and Viticulture 16:144-158.

Smirnova G., Z. Samoilova, N. Muzyka and 0. Oktyabrsky (2012) Influence of plant polyphenols and medicinal plant extracts on antibiotic susceptibility of Escherichia coli. Journal of Applied Microbiology 113:192-199

Sroka Z. and W. Cisowski (2003) Hydrogen peroxide scavenging and antiradical activity of some phenolic acids. Food and Chemical Toxicology 41:753-758.

Taiz L. and E. Zeiger (2002) Plant Physiology. $3^{\text {rd }}$ ed. Sinauer Associates, Inc. Sunderland, Massachussetts, USA. $690 \mathrm{p}$

Tarchoune I., C. Sgherri, R. Izzo, M. Lachaal, Z. Ouerghi and F. Navari-Izzo (2010) Antioxidative responses of Ocimum basilicum to sodium chloride or sodium sulphate salinization. Plant Physiology and Biochemistry 48:772-777

Tarchoune I., C. Sgherri, R. Izzo, M. Lachaâl, F. Navari-Izzo and Z. Ouerghi (2012) Changes in the antioxidative systems of Ocimum basilicum L. (cv. Fine) under different sodium salts. Acta Physiologiae Plantarum 34:1873-1881.

Tarchoune I., O. Baâtour, J. Harrathi, P. L. Cioni, M. Lachaâl, G. Flamini and Z. Ouerghi (2013) Essential oil and volatile emissions of basil (Ocimum basilicum) leaves exposed to $\mathrm{NaCl}$ or $\mathrm{Na}_{2} \mathrm{SO}_{4}$ salinity. Journal of Plant Nutrition and Soil Science 176:748-755.

Toussaint J. P., F. A. Smith and S. E. Smith (2007) Arbuscular mycorrhizal fungi can induce the production of phytochemicals in sweet basil irrespective of phosphorus nutrition. Mycorrhiza 17:291-297.

Witzell J., R. Gref and T. Nasholm (2003) Plant-part specific and temporal variation in phenolic compounds of boreal bilberry (Vaccinium myrtillus) plants. Biochemical Systematics and Ecology 31:115-127.

Zhang Q., S. Wu, C. Chen, L. Z. Shu, X. J. Zhou and S. N. Zhu (2014) Regulation of nitrogen forms on growth of eggplant under partial rootzone irrigation. Agricultural Water Management 142:56-65.

Zulfugarov I. S., A. Tovuu, J. H. Kim and C. H. Lee (2011) Detection of reactive oxygen species in higher plants. Journal of Plant Biology 54:351-357. 